\title{
The Barbed Suture in Bowel Stapled Anastomoses
}

\section{Fabio Pacifico*}

Ospedale Fatebenefratelli, Benevento, BN Italy

\begin{abstract}
The authors provide a review of self-anchoring suture with unidirectional fins, called "barbed suture", that is an important technological innovation in surgery over the last decade. In their early experience the Authors used the V-Loc device in skin suture, then in urology, and only recently in intestinal surgery. They describe a technique for closure of the enterotomy after mechanical bowel side-to-side anastomosis using the "barbed sutures." The Authors report their 66 sutures experience in different intestinal segments anastomoses. The procedure was safe and reproducible with good results.
\end{abstract}

Keywords:Barbed suture; Bowel anastomoses

\section{Introduction}

To-day the mechanical bowel side-to-side anastomosis is recommended for many surgical procedures, especially for ileocolic one, after right hemicolectomy [1]. This type of anastomosis requires a dual enterotomy for the stapler access. The closing of the enterotomies is currently performed with different methods and sutures. The use in bowel surgery of self-retaining wire with fins, so-called "barbed suture", has been recently described [2,3]. It is a knotless unidirectional barbs wire of absorbable polygluconate, dioxanone and trimethylene carbonate: it's absorbable in 90 days $\left(\mathrm{V}-90 \operatorname{loc}^{\mathrm{TM}}\right.$, Covidien ${ }^{\mathrm{TM}}$, Mansifield, MA, USA). The Authors performed the enterotomy suture with the "barbed sutures" both in open and in laparoscopic surgery as described below. They evaluated the results obtained.

\section{Material and Methods}

After an initial period where we have used the "barbed sutures" in urological laparoscopic surgery, we considered the use of the same in intestinal surgery. In 24 months were enrolled 58 consecutive patients laparoscopic and not without exclusion criteria. In these cases, the unidirectional "barbed suture" has been employed for the closure of the enterotomy after mechanical side-to-side isoperistaltic anastomosis. In 3 patients, one operated in laparoscopy and two in open surgery, with gastric resection for adenocarcinoma, the wire with fins has been employed for the closure of the enterotomy both in gastro-jejunal that the jejunal-jejunal. In another patient always two sutures after pancreaticoduodenectomy. In 25 patients (6 laparoscopic and 19 open) the closure of the opening was carried out in the ileocolonic isoperistaltic anastomosis after right hemicolectomy. In an additional 29 patients, all operated in open surgery, the ileo-ileal anastomosis was always isoperistaltic.

\section{Surgical Technique}

The technical aspects of the suture are the use of two unidirectional absorbable "barbed suture". The wire used was a $3-0 / 15 \mathrm{~cm}$ in laparoscopy and $3-0 / 30 \mathrm{~cm}$ in open surgery with $1 / 2$ circle $/ 26 \mathrm{~mm}$ needle. The suture starts from the corner of opening with the inside-out and then outsidein passage (Figure 1a). The needle is then inserted inside the eyelet and the continuous suture approaches the layers serosa-muscle from both sides until mid gap always with passages inside-out and outside-in. You run the same route from the opposite corner with another wire. The two sutures are joined in the middle of the gap without making knots (Figure 1b). The described mode allows a secure suture of both corners.

\section{Results}

The procedures were performed by two experienced surgeons both in laparoscopy and in open surgery. The mechanical anastomosis was



performed with different staplers at random. Patients were 68 years old age (range 56-88) with ASA score mainly II-III: the most comorbidities were heart and/or pulmonary disease and diabetes. The wire has proven very handy with good smoothness and continuous tension. We have performed a total of 66 sutures of the intestinal openings after mechanical anastomosis in 58 enrolled patients. The procedures included 20 sutures performed in urgency for bowel obstruction and two for gut perforation. There were no complications related to the

*Corresponding author: Pacifico F, MD, Ospedale Fatebenefratelli, Benevento, BN Italy, Tel: +393932618831 E-mail: fabiopacifico@libero.it

Received February 02, 2015; Accepted February 28, 2015; PublishedFebruary 10, 2014

Citation: Pacifico F, Fatebenefratelli O, Benevento, Italy BN (2014) The Barbed Suture in Bowel Stapled Anastomoses Surgery Curr Res 5: 219. doi:10.4172/21611076.1000219

Copyright: (๑) 2014 Pacifico F, et al. This is an open-access article distributed under the terms of the Creative Commons Attribution License, which permits unrestricted use, distribution, and reproduction in any medium, provided the original author and source are credited. 




Figure 2: The second suture starts from the opposite corner.

closure of the enterotomy, as filtration or postoperative dehiscence.

\section{Discussion}

The mechanical intestinal isoperistaltic side-to-side anastomosis is used to-day in different bowel segments. This type of anastomosis was studied and compared to others especially in Crohn' disease surgery, where it seems to have dehiscence rates lower than the end-to-end or the side-to-end anastomosis [4]. Therefore, to-day both in elective and in emergency surgery it would be better to use the side-to-side anastomosis, especially in the ileocolic anastomosis (1 A evidence level), the ileo-ileal and gastro-ileal ones [1]. This type of suture allows the opposition of the terminal bowel stumps without tension, and then the isoperistaltic alignment promotes the intestinal transit. The closure of residual intestinal openings for the insertion of the stapler is carried out with different techniques and sutures. In most cases it's possible to perform a single-layer muscle-serosa running suture: in fact the visceral peritoneum is an excellent anastomosis seal more and more than muscle [5]. A greater caution should be paid to two created corners. Therefore, the Authors recommend the use of two wires starting really from the corners: the suture with the wire wing requires maximum traction at each passage. A running self-anchoring suture, narrowing the lumen in proceeding little by little, would not allow a good view of the opposite corner. In laparoscopy it is certainly advantageous to have a knotless and self-anchoring wire like "barbed sutures ". The good results in Laparoscopy have led the Authors to use it in open surgery. Therefore they evaluated the efficacy of "barbed suture "for closing enterotomies both in laparoscopy and in open surgery: the results are encouraging. The early experience with the "barbed suture" in laparoscopic bowel surgery has been newly described by the Luxembourg group of Azagra et al., the data encourage its use not only for closing enterotomies but also for the entire anastomosis [3]. Authors' starting trend confirms all that. The procedure is safe and reproducible and offers warranty of sealing like other sutures. Furthermore, the "barbed suture" allows eliminating the drawbacks related to the node and to the uniformity of tension of the running suture that requires expert hands in laparoscopy. References

1. Goulder F (2012) Bowel anastomoses: The theory, the practice and the evidence base. World J Gastrointest Surg 4: 208-213.

2. Nemecek E, Negrin L, Beran C, Nemecek R, Hollinsky C (2013) The application of the V-loc closure device for gastrointestinal sutures: a preliminary study. Surg Endosc 27: 3830-3834

3. Facy O, De Blasi V, Goergen M, Arru L, De Magistris L, et al. (2013) Laparoscopic gastrointestinal anastomoses using Knotless barbed suture are safe and reproducible: a single centre experience with 201 patients. Surg Endosc 27: 3841-3845.

4. Resegotti A, Astegiano M, Farina EC, Ciccone G, Avagnina G, et al. (2005) Side-to-side stapled anastomosis strongly reduces anastomotic leak rates in Crohn's disease surgery. Dis Colon Rectum 48: 464-468.

5. Chen C (2012) The art of bowel anastomosis. Scandinavian J Surg 101 238-240. 